\title{
Search for an Optical Counterpart of PSR J0537-6910
}

\author{
Christian Gouiffès \\ Service d'Astrophysique, CEA/DSM/DAPNIA, C.E. Saclay, 91191 \\ Gif-sur-Yvette Cedex, FRANCE, email: cgouiffes@cea.fr
}

\section{Hakki Ögelman}

Department of Physics, University of Wisconsin, Madison, WI 53706, U.S.A , email: ogelman@astrog.physics.wisc.edu

\begin{abstract}
We have conducted an optical study of the recently discovered very fast ( $\mathrm{P} \sim 16 \mathrm{msec}) \mathrm{X}$-ray pulsar PSR J0537-6910 in the supernova remnant $\mathrm{N} 157 \mathrm{~B}$. Broad band imaging has been obtained to study the field around the $\mathrm{X}$-ray position and a high speed photometry programme was carried out in an attempt to detect optical pulses.
\end{abstract}

\section{Introduction}

The recent detection in the X-rays band of a fast pulsar inside the supernova remnant SNR 0539-69.1 (N157B) confirms the association of this SNR with the class of the young Crabe-like supernova remnants where the synchrotron emission is powered by a central young pulsar. The pulsar was first detected in the 2-10 KeV band with RXTE and ASCA (Marshall et al. 1998). While the pulsar has not yet be detected in the radio band (Crawford et al. 1998), Wang and Gotthelf (1998), using a deep ROSAT HRI observation, were able to localize the pulsar with a good spatial accuracy. A search for an optical emission (pulsed or not) was then undertaken.

\section{Observations and Analysis}

We have obtained $B$ and $V$ exposures on the field of $N 157 B$ using the 1.54 Danish telescope (1.54D) at La Silla and a R-band image taken under good seeing condition at the New Technology Telescope (NTT) equipped with SUSI2 on 1999, January 10. Standard astrometric and photometric analysis were performed. High Speed Photometry on the field was carried out at the $3.6 \mathrm{~m}$ telescope equipped with the fast photometer two periods: 1991, November and 1992, January. Briefly the instrument consists in a single channel photometer coupled with a photomultiplier sampling the data at $10 \mathrm{kHz}$ (see Ögelman et al. 1990, Gouiffes et al. 1992 for more informations). The diaphragm $\left(10^{\prime \prime}\right)$ was centered on star number 2 (figure 1) and no filter was used. After the barycentric corrections the data were folded around the extrapolated $\mathrm{X}$-rays frequency since the existence of glitches has been mentioned (Marshall, F.E. these proceedings).

\footnotetext{
${ }^{1}$ Based on data collected at the European Southern Observatory, La Silla, Chile.
} 


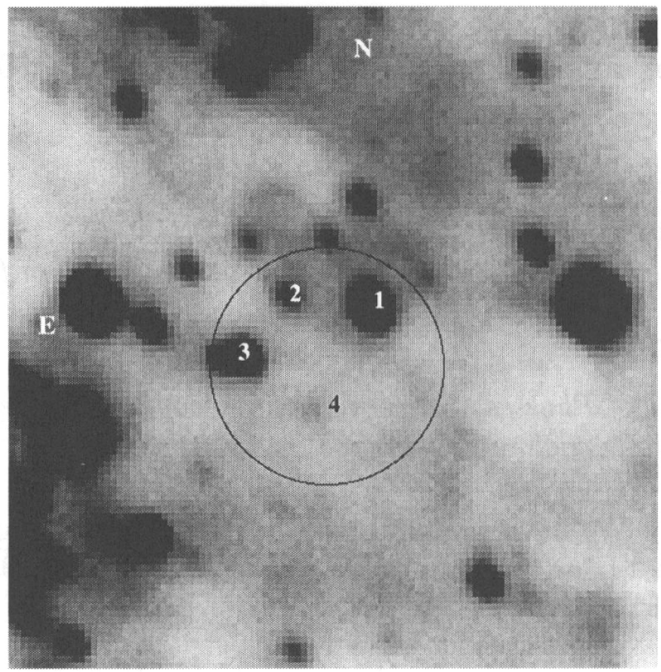

Figure 1. R-band image of the field around PSR J0537-6910.

\section{Results}

The figure 1 shows the region around the ROSAT position (circle of $3^{\prime \prime}$ radius) of PSR J0537-6910 as extracted from our R-band NTT image. Several objects labeled 1,2, 3 and 4 (with respectively R-mag of 18.6,21.7, 18.9 and 22.3) are inside the error box but are very unlikely the optical counterpart. In fact our more stringent limit comes from our fast photometry analysis where no signal has been detected in none of our data sets. Based on several simulations to evaluate our sensitivity and contemporaneous observations of PSR B0540-69 ( $\mathrm{m}_{V} \sim 22.4$ ) made with the same instrumental configuration, we could set a relative conservative upper limit of $\mathrm{m}_{V} \sim 23$ on this first tentative to detect optical pulses from PSR J0537-6910. Deeper planned optical observations (associated with an improved X-ray position) will certainly bring more constraints on the optical emission of this fast and young pulsar.

Acknowledgments. It is a pleasure to thank Thomas Augusteijn and Eric Pantin for acquiring the CCD images.

\section{References}

Crawford, F. \& al. 1998, MmSAI, 69, 959

Gouiffes, C. \& al. 1992, ApJ, 394, 581

Marshall, F.E. \& al. 1998 ApJ, 499, L179-L182

Wang, Q.D. \& Gotthelf, E. V. 1998, ApJ, 494, 623-635

Ögelman, H. \& al 1990, A\&A, 237, L90 